\title{
Safety and Efficacy of Stent Graft Implantation for Malignant Superior Vena Cava Syndrome
}

\author{
Shoujin Cao, MD, Yinghua Zou, MD, Tianshi Lyu, MD, Zeyang Fan, MD, Haitao Guan, MD, Li Song, MD, \\ Xiaoqiang Tong, MD, Jian Wang, MD
}

Department of Interventional and Vascular Surgery, Peking University First Hospital, Beijing, China

\section{ABSTRACT}

Background: Patients with malignant superior vena cava syndrome (SVCS) usually require urgent treatments due to a high potential risk of early mortality. Stent implantation can rapidly improve the symptoms of SVCS, which may be beneficial to subsequent anti-tumor therapy. The aim of the study was to evaluate the clinical outcomes of stent graft implantation for the treatment of superior vena cava (SVC) obstruction caused by non-small cell lung carcinoma (NSCLC) with acute post-stenting occlusion.

Methods: Between October 2014 and December 2019, 16 patients were selected for stent graft implantation. Technical success and clinical efficacy were assessed. Stent patency and patient survival rates, as well as the complications were analyzed.

Results: There were 17 stent grafts implanted in 16 patients. The technical success was $100 \%$. The residual stenosis after initial implantation was $64.0 \pm 9.0 \%$. The stent expanded to an optimal size in $5.5 \pm 2.2$ days after the initial deployment. Migration occurred when deploying of the stent graft in one patient; this stent graft was successfully stabilized by a second one. No other complications related to the procedure were found except one migration. At 1, 3, 6, 9 and 12 months, the cumulative survival rates were 100\%, 75\%, 56\%, $19 \%$ and $0 \%$, respectively. The mean OS was 173 days. The median survival was 166 days.

Conclusions: Stent graft can be safely used in patients with SVC obstruction with a good long-term patency rate.

\section{INTRODUCTION}

Endovascular stent implantation has been an acceptable palliative treatment for patients with malignant SVC syndrome [Rowell 2002]. Bare stent has been investigated and proved to be a reliable relief from the symptoms caused by SVC obstruction. Studies also showed the acute occlusion of bare stent could be encountered, due to an immediate

Received fuly 13, 2021; accepted August 13, 2021.

Correspondence: Dr. Fian Wang, Department of Interventional and Vascular Surgery, Peking University First Hospital, No. 8 Xishiku Street, Xicheng District, Beijing, China; +8610-83572633; fax: +8610-83572633 (e-mail: jianwang0987@sina.com). penetration of the tumor thrombus to the stent lumen, this was frequently seen in patients with local unstable tumor thrombus (Figure 1). Even with an initial successful placement of a bare stent, the recurrence of the symptoms also occurs in up to $41 \%$ of patients, this has been attributed to tumor growth inside the stent or venous thrombosis [Armstrong 1987; Mose 2006; Dyet 1993]. A few reports [Gill 2000; Azizzadeh 2007; Mansour 2009] described the use of covered stents in recurrent SVC syndrome after bare stent placement or iatrogenic injury of the SVC. In this study, we report our primary experiences and evaluate the outcomes of stent-graft implantation

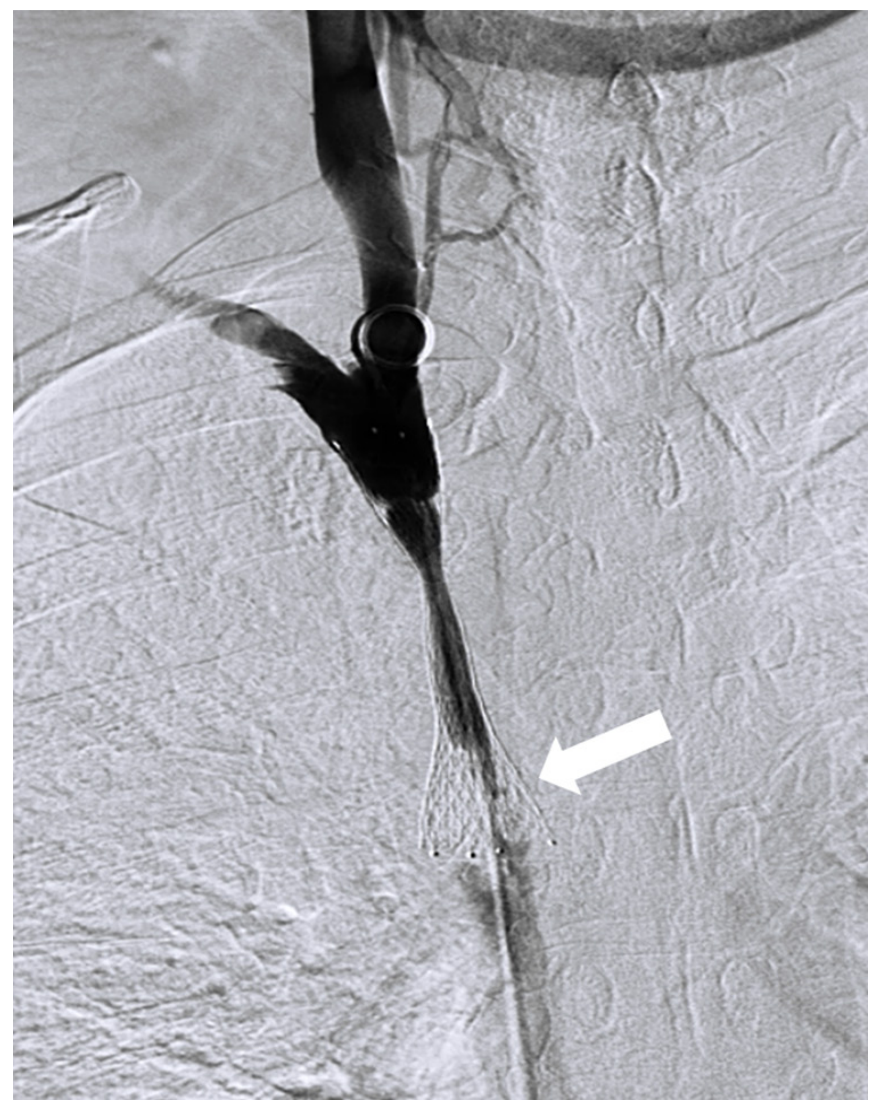

Figure 1. Acute occlusion of a bare stent after deployment in a severe stenotic SVC. Angiography showed filling defects in the distal portion of the stent (white arrow), which was thought to be the tumor thrombus protruding into the lumen from the mesh of the stent. 
Table 1. Baseline characteristics of the study

\begin{tabular}{|c|c|}
\hline Parameter & $\mathrm{N}$ \\
\hline Sex (no. of patients) & 16 \\
\hline $\mathrm{F}$ & 6 \\
\hline M & 10 \\
\hline \multicolumn{2}{|l|}{ Age $(y)$} \\
\hline Mean \pm SD & $65.4 \pm 6.4$ \\
\hline Range & $53-78$ \\
\hline \multicolumn{2}{|c|}{ Cause of SVC obstruction (no. of patients) } \\
\hline NSCLC & 16 \\
\hline Stage IIIA & 1 \\
\hline Stage IIIB & 3 \\
\hline Stage IIIC & 4 \\
\hline Stage IV & 8 \\
\hline \multicolumn{2}{|c|}{ Length of SVC obstruction (mm) } \\
\hline Mean \pm SD & $51.8 \pm 10.0$ \\
\hline Range & $36-73$ \\
\hline \multicolumn{2}{|c|}{ Treatment before stent graft placement (no. of patients) } \\
\hline None & 1 \\
\hline RT & 1 \\
\hline Chemotherapy & 14 \\
\hline Chemotherapy and RT & 0 \\
\hline Surgery & 0 \\
\hline \multicolumn{2}{|c|}{ Treatment of stent graft placement (no. of patients) } \\
\hline None & 0 \\
\hline RT & 5 \\
\hline Chemotherapy & 11 \\
\hline Chemotherapy and RT & 0 \\
\hline
\end{tabular}

NSCLC, non-small cell lung cancer; RT, radiotherapy; SVC, superior vena cava

in patients suffered from malignant SVC syndrome with the local lesion of high risk of acute post-bare-stenting occlusion.

\section{MATERIALS AND METHODS}

The study was approved by the institutional review board, and written informed consent was obtained from each patient or from his or her legal guardian. Between October 2010 and December 2019, 16 patients (six women and 10 men; mean age, $65.4 \pm 6.4 \mathrm{y}$; range, 53-78 y) with stage IIIA (one patient), IIIB (three patients), IIIC (four patients), and IV (eight patients) NSCLC and SVC obstruction were identified and selected for stent graft implantation. The criterions for high risk of post-bare-stenting occlusion in this study was based on the following manifestations on enhanced CT scan: (1)Local lesion with a stenotic rate $\geq 90 \%$, length $\geq 3 \mathrm{~cm}$; (2) Eccentric and irregular boarder of the stenotic lesion; (3) An abrupt
Table 2. Symptoms of SVC obstruction, according to Kishi et al [Sacks 2003]

\begin{tabular}{ll}
\hline Symptoms & $\mathrm{N}$ \\
\hline Neurologic symptoms & 2 \\
$\quad$ Stupor, coma, or blackout & 8 \\
Blurry vision, headache, dizziness, or amnesia & 2 \\
Changes in mentation & 1 \\
Uneasiness & \\
Laryngopharyngeal or thoracic symptoms & 2 \\
Orthopnea or laryngeal edema & 2 \\
Stridor, hoarseness, dysphagia, glossal edema & 14 \\
Shortness of breath & 8 \\
Cough or pleural effusion & \\
Nasal and facial signs or symptoms & 2 \\
Lip edema, nasal stiffness, epistaxis, or rhinorrhea & 16 \\
Facial swelling & \\
Venous dilatation & 16 \\
Neck vein or arm vein distention, upper extremity & \\
swelling, or upper body plethora & \\
\hline
\end{tabular}

SVC, superior vena cava

margin from the normal SVC lumen. Patients who had the image characteristics of (1)+(2)+(3) were selected and listed in Table 1. All identified patients were treated with implantation of stent graft (Fluency, Bard, USA), and no other stents were implanted during this period. Clinical symptoms were noted, according to Kishi et al [Sacks 2003] and were shown in Table 2. Improvements in the clinical symptoms were evaluated according to the International Consensus Committee on Chronic Venous Disease [Yuuki 2001].

\section{Stent implantation}

For all patients, a combined right-basilic vein and right femoral vein approach were established under local anesthesia. Short 5-F sheath was placed in the right basilic vein and right femoral vein. From the femoral approach, a 5-F pigtail catheter was placed proximally to the SVC stenosis, a single curved angiographic catheter was introduced from the right basilic approach and placed at the distal portion of the SVC obstruction. Venography was performed simultaneously via the single curved angiographic catheter and the pigtail catheter to obtain a well delineation of the whole morphology of the lesion (Figure 2). Recanalization of the obstruction was achieved by using a hydrophilic guide wire (Terumo, Tokyo, Japan) and a 5-F catheter (Glidecath; Terumo) via the femoral approach. After the obstruction was crossed, a super-stiff guide wire (Amplatz Super Stiff Guidewire; Boston Scientific) was placed across the stenosis. Followed by introducing of a $65 \mathrm{~cm}, 10-\mathrm{F}$ sheath (Super Arrow-Flex; Arrow International, Inc, Reading, Pennsylvania), the stent-graft was released. Road map guidance was performed via the single curved angiographic catheter inside the right 


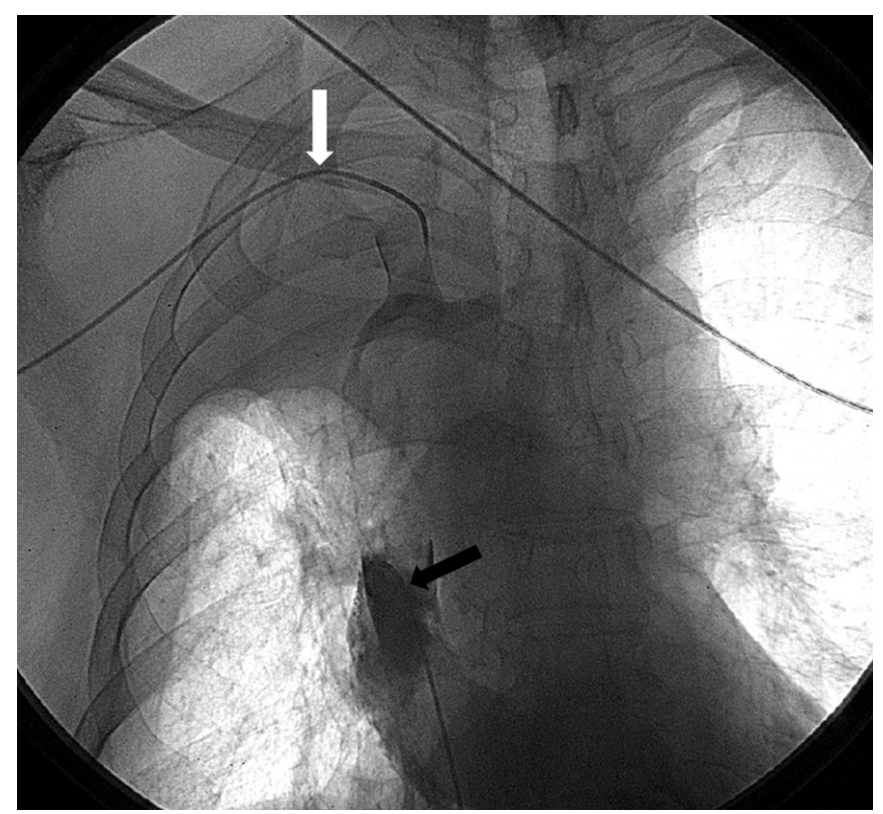

Figure 2. Angiography simultaneously from the single curve catheter (with arrow) and the pig tail catheter (black arrow) introduced from the right basilic vein and the right femoral vein, respectively, can clearly delineate the whole length of the severe SVC obstruction.

subclavian vein to control adequate stent positioning during the who deploying procedure (Figure 3). Technical success in this study was defined as the complete coverage of the stenosis (stent overlapping the margins of the stenosis by at least 1 $\mathrm{cm}$ on either side). No additional percutaneous transluminal angioplasty (PTA) was performed after stent graft implantation for this study group. All patients received 5,000 IU of heparin during the intervention and anti-coagulation with low-molecular-weight heparin for two weeks after the procedure.

\section{Follow up}

Data regarding the technical aspects of stent placement and procedure and device-related complications were recorded. Chest film was taken every day to identify the morphological changes of the stent graft after the deployment. To evaluate the symptom improvement and patient survival, all patients were interviewed by telephone at weekly intervals. All patients were followed up for checking of the recurrence of symptoms of SVC syndrome until death. Stent patency was monitored by enhanced CT scan performed at 1, 3, 6, 9, and every three months after the initial procedures. Patients who underwent chemotherapy and/or radiation therapy after stent placement were also evaluated with enhanced CT of the chest during the course and/or end of their therapies.

\section{Clinical outcomes, study endpoints, and statistical analysis}

Clinical outcomes were evaluated, according to the International Consensus Committee on Chronic Venous Disease [Azizzadeh 2007]. Additional therapies after stent implantation were noted. Complications were classified as major or minor, according to the guidelines of the

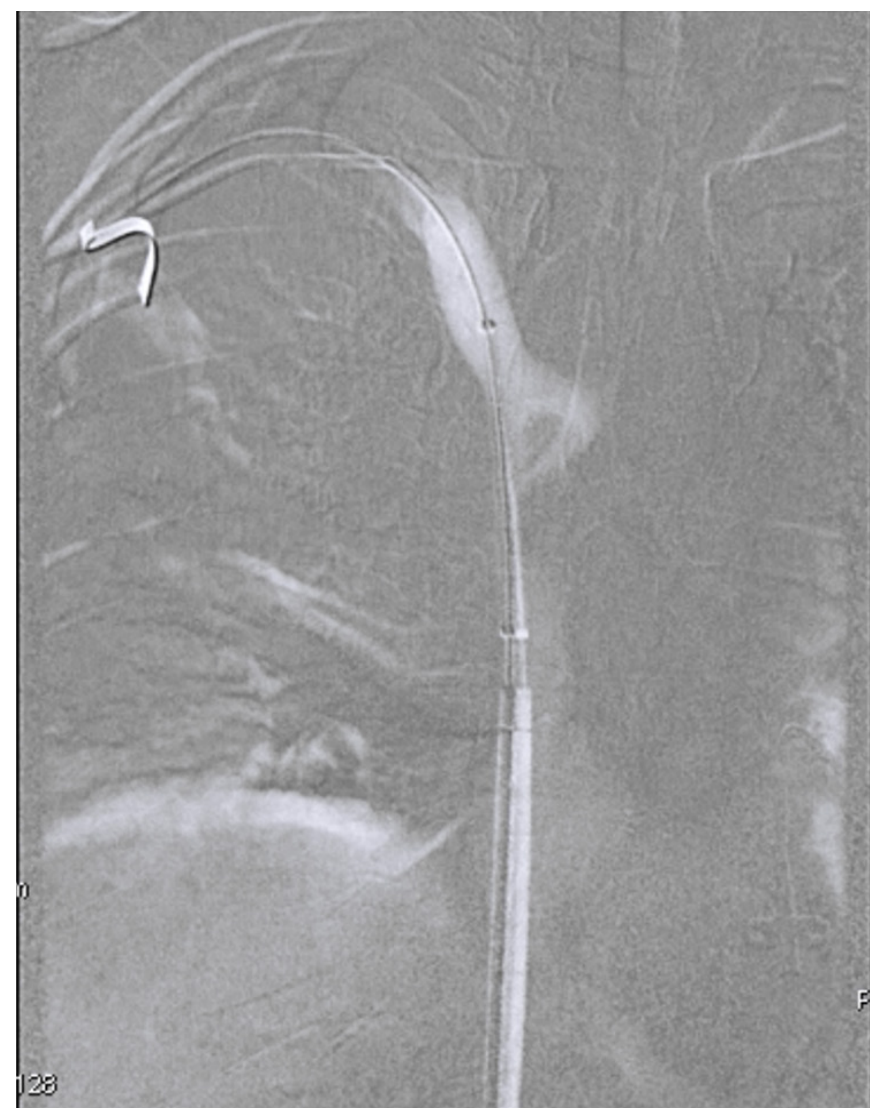

Figure 3. Road map image was performed from the single curve catheter introduced from the right basilic vein to assist the deployment of the stent graft.

Society of Interventional Radiology Standards of Practice Committee [Porter 1995]. We defined major complications as complications that necessitated major therapy or an unplanned increase in the level of care or prolonged hospitalization, and those that resulted in permanent adverse sequelae or death. We defined minor complications as complications that required nominal or no therapy. The primary study endpoints were technical success, which was defined as accurate stent placement with complete coverage of the obstructed SVC, and clinical efficacy. Secondary study endpoints were periprocedural complications. All data were recorded in a database for descriptive statistics including ranges. These statistical procedures were performed using SPSS software (Version 20.00; GraphPad Software, La Jolla, California).

\section{RESULTS}

\section{Technical and clinical outcomes}

Stent graft placement was technically successful in all 16 patients. We choose stent graft with diameter $13.5 \mathrm{~mm}$ for all the patients. The residual stenosis rate after initial stent graft placement was $64.0 \pm 9.0 \%$ (range $45 \%-84 \%$ ). The stent expanded to an optimal open size (defined as residual stenosis 


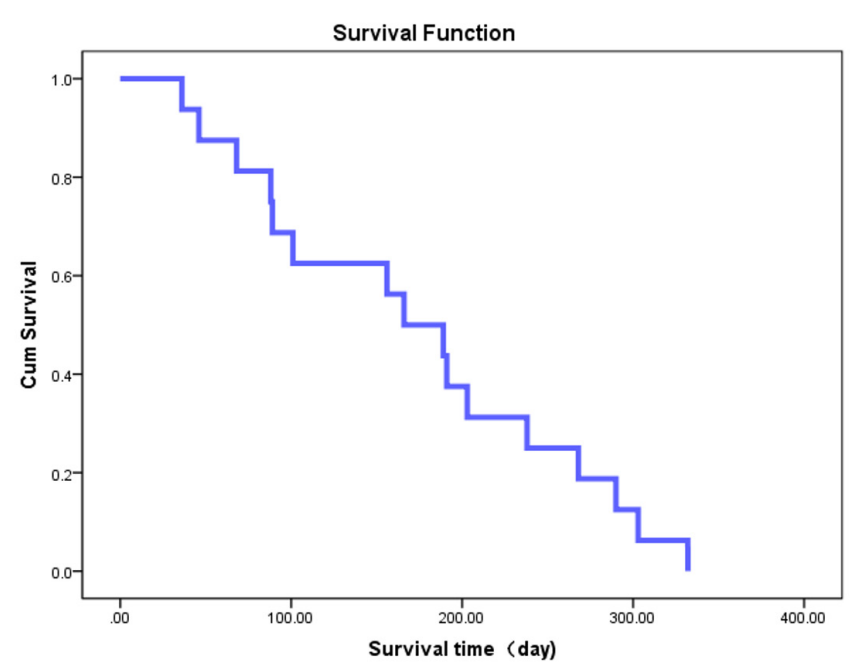

Figure 4. Kaplan-Meier curves of this study group: The cumulative survival rates were $100 \%, 75 \%, 56 \%, 19 \%$ and $0 \%$ at $1,3,6,9$ and 12 months, respectively. The median survival was 166 days ( $95 \%$ confidence interval: 101, 231 days).

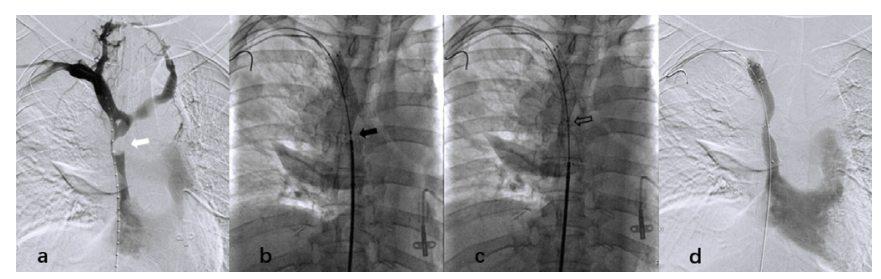

Figure 5. Migration of the stent graft in a patient with SVC obstruction. (a) Angiography showed a sever stenosis of the SVC because of the invasion tumor (with arrow). (b) The first stent graft was moving front when deploying (black arrow). (c) The second stent graft was deployed crossed the stenosis and stabilized the first one (hollow arrow). (d) angiography showed recanalization of SVC with an improved blood flow.

$<10 \%$ ) in $5.5 \pm 2.2$ days (range 2-10 days) after the initial deployment. In order to avoid stent migration, no post PTA for stent graft was performed after the deployment. The clinical symptoms improved in all 16 patients $(100 \%)$. The mean symptom relieve time was $3.3 \pm 1.4$ days (range 1-6 days). We found no procedure-related complications or mortality in this group.

\section{Patient survival and stent graft patency}

The mean clinical follow up was $172.8 \pm 95.3$ days (range, 36-332 days). All patients died at the end of follow up. Kaplan-Meier analysis revealed that the cumulative patency was $100 \%$ at $1,3,6$, and 9 months. At 1, 3, 6, 9 and 12 months, the cumulative survival rates were $100 \%, 75 \%$, $56 \%, 19 \%$ and $0 \%$, respectively. The mean OS was 173 days (95\% confidence interval: 126, 219 days). The median survival was 166 days (95\% confidence interval: 101, 231 days) in this study group. (Figure 4) During the follow-up period for whole patients, no clinical signs of symptom recurrence caused by SVC restenosis were found. Follow up enhanced CT scan also showed $100 \%$ patency rate at 1, 3, 6, 9 months

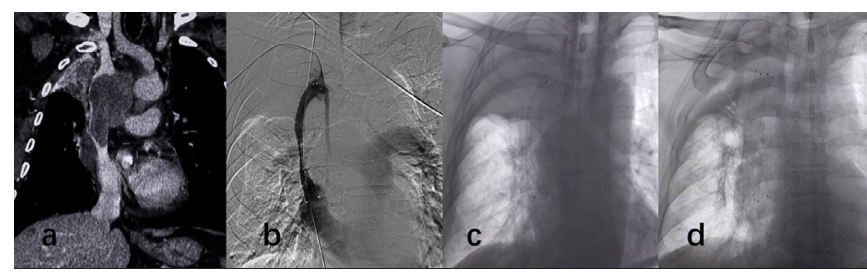

Figure 6. Stent graft implantation for a patient with SVC obstruction. (a) Enhanced CT scan showed the whole morphology of the SVC obstruction. (b) Angiography showed an improved blood flow in SVC, post PTA was not performed during the procedure. (c) Chest film showed an $80 \%$ residual stenosis of the stent graft after the initial procedure. (d) Angiography showed the stent graft fully opened 10 days after the initial procedure, and the symptoms of the patient were remarkably improved, accordingly.

after initial stent graft placement. The longest follow-up time was 332 days for one patient before death event, this patient died from cerebral metastasis and showed no evidence of SVC restenosis after the initial stent graft placement.

\section{Complication}

Except for one stent graft migration, no other major or minor complications were recorded in this study. The stent migration was opposite to the heart direction because of the front moving of the stent graft, when releasing it from the delivery system. This stent graft was then stabilized by anther stent graft (Figure 5).

\section{DISCUSSION}

SVC occlusion is a severe clinical circumstance that leads to disastrous results. The standard treatment of SVC occlusion is the recanalization by the deployment of the stent [Angle 2010]. Bare stent is often used with a relatively good clinical result. Restenosis or occlusion of the stent by tumor ingrowth or thrombus formation is the main drawback of the bare stent, which affects the longer-term patency [Kunal 2019]. Acute occlusion of bare stent could even happen if an unstable lesion was presented and could easily penetrate the mesh of the bare stent and protrude into the stent lumen [Salavitabar 2018]. Pulmonary embolization is another severe complication when bare stent is deploying. Stent graft might be a better choice for a certain kind of lesion which could have a high risk of acute occlusion rate [Haddad 2018].

The reason we choose stent graft for this study group is the special characteristics of the SVC lesion on the enhanced CT scan. A wide boundary protruding lesion with an abrupt margin in a severe stenotic SVC lumen on CT scan always suggests an unstable tumor thrombus, which may penetrate into SVC lumen from the mesh of a deployed bare stent and may result in an acute obstruction of the SVC stent. When this acute occlusion happens, pulmonary embolization might even occur [Volpi 2018]. The best advantage of stent grafts is prevention of an acute occlusion of the SVC. The disadvantage is migration of the stent grafts because of the smooth 
surface of it [Kuo 2017]. The maximum stent graft that can be used in our medical center is $13.5 \mathrm{~mm}$ in diameter, so in this study group, we chose only lesions with stenotic rate $\geq 90 \%$ and length $\geq 3 \mathrm{~cm}$ to be treated with the stent graft; the reason was to avoid the migration because of the relatively small size of the stent graft. This kind of lesion has a higher incidence of acute occlusion when treated with a bare stent, so we did not set up a compare group [Wang 2020]. The other advantage of the stent graft is the long patency rate, in our study, the 1, 3, 6,9 month's patency was $100 \%$, no patients suffered from the SVC syndrome again after deployment of stent graft.

In addition, because of the severe stenotic rate, we did the angiography from both sides of the SVC lesion simultaneously to delineate the detailed morphology of the lesion. The real time road-map guidance could also be performed by the single curved catheter from the right basilic vein approach for the accurate localization. The position of the stent graft then could be fully understood during the whole deploying procedure. In this study, the residual stenosis of the SVC immediately after stent graft deployment was $64.0 \pm 9.0 \%$ (range $45 \%-84 \%$ ), we did not perform the post dilatation because we believed that it was safer for the stent graft to gradually expand by itself. We also did not measure the pressure gradient across the stenosis because we thought it could be omitted if the post-stent PTA was not intended. We found that all the residual stenosis were improved $5.5 \pm 2.2$ days (range 2-10 days) after the stent graft deployment (Figure 6). Clinical symptoms were improved after stent graft placement. The time for symptom relief was $3.3 \pm 1.4$ days (range 1-6 days); the longest time of symptom relief was 6 days after stent graft placement. The reason for delayed symptom relief was thought to be gradual expansion of the stent graft, which may result in gradual improvement of the blood flow, accordingly.

The occurrence of SVCS can rapidly reduce the quality of each patient's remaining life and dramatically increase the risk of early mortality [Ampil 2018]. Although stent insertion has no effect on anti-tumor, good clinical condition of patients after stent insertion could provide the prolonging anti-tumor treatment, which can prolong patient survival [Xu 2015]. In this study, all patients underwent subsequent anticancer treatment after stent insertion, due to rapid relief of symptoms of SVCS. The median survival was 166 days (95\% confidence interval: 101, 231 days) in this study group, which was similar to results of previous reports [Wang 2020; Niu 2017]. And all the patients in this study died from tumorrelated complications.

In summarization, stent graft safely can be used for patients suffering from SVC obstruction with good longterm patency. Improvement of the clinical symptoms might be delayed because of gradual expansion of the stent graft to an adequate level of normal hemodynamics. To decrease the risk of migration, proper selection of patients should be emphasized. Post-stent graft PTA should be avoided.

The main shortcoming of this study was the relatively small size of the study group. Also, clinical intervention to further prolong patient OS should be expected.

\section{REFERENCES}

Ampil FL, Caldito G, Devarakonda S, Vora M, Mills G, Milligan S. 2018. Longevity after radiotherapy of stage III lung cancer: superior vena cava obstruction is associated with early mortality. Ann Palliat Med. 7(2): 242-8.

Angle JF, Siddiqi NH, Wallace MJ, et al. 2010. Quality improvement guidelines for percutaneous transcatheter embolization: Society of Interventional Radiology Standards of Practice Committee. J Vasc Interv Radiol. 21(10): 1479-86.

Armstrong BA, Perez CA, Simpson JR, Hederman MA. 1987. Role of irradiation in the management of superior vena cava syndrome. Int $\mathrm{J}$ Radiat Oncol Biol Phys. 13(4): 531-9.

Azizzadeh A, Pham MT, Estrera AL, Coogan SM, Safi HJ. 2007. Endovascular repair of an iatrogenic superior vena caval injury: a case report. J Vasc Surg. 46(3): 569-71.

Dyet JF, Nicholson AA, Cook AM. 1993. The Use of the Wallstent Endovascular Prosthesis in the Treatment of Malignant Obstruction of the Superior Vena-Cava. Clin Radiol. 48(6): 381-5.

Gill K, Ettles DF, Nicholson AA. 2000. Recurrent superior vena caval obstruction due to invasion by malignant thymoma: treatment using a stent-graft. Brit J Radiol. 73(873): 1015-7.

Haddad MM, Simmons B, McPhail IR, et al. 2018. Comparison of Covered Versus Uncovered Stents for Benign Superior Vena Cava (SVC) Obstruction. Cardiovasc Intervent Radiol. 41(5): 712-7.

Kunal S, Pathak V, Pathak P. 2019. Superior vena cava syndrome due to pacemaker leads: An Indian report with a review of management practice. Monaldi Arch Chest D. 89(1).

Kuo TT, Chen PL, Shih CC, Chen IM. 2017. Endovascular stenting for end-stage lung cancer patients with superior vena cava syndrome post first-line treatments - A single-center experience and literature review. J Chin Med Assoc. 80(8): 482-6.

Mansour M, Altenburg A, Haage P. 2009. Successful emergency stent implantation for superior vena cava perforation during malignant stenosis venoplasty. Cardiovasc Intervent Radiol. 32(6): 1312-6.

Mose S, Stabik C, Eberlein K, Ramm U, Bottcher HD, Budischewski K. 2006. Retrospective analysis of the superior vena cava syndrome in irradiated cancer patients. Anticancer Res. 26(6C): 4933-6.

Niu S, Xu YS, Cheng L, Cao C. 2017. Stent insertion for malignant superior vena cava syndrome: effectiveness and long-term outcome. Radiol Med. 122(8): 633-8

Porter JM, Moneta GL. 1995. Reporting standards in venous disease: an update. International Consensus Committee on Chronic Venous Disease. J Vasc Surg. 21(4): 635-45.

Rowell NP, Gleeson FV. 2002. Steroids, radiotherapy, chemotherapy and stents for superior vena caval obstruction in carcinoma of the bronchus: a systematic review. Clin Oncol (R Coll Radiol). 14(5): 338-51.

Sacks D, McClenny TE, Cardella JF, Lewis CA. 2003. Society of Interventional Radiology clinical practice guidelines. J Vasc Interv Radiol. 14(9 Pt 2): S199-202

Salavitabar A, Flyer JN, Torres AJ, et al. 2018. Transcatheter stenting of superior vena cava obstruction after pediatric heart transplantation: A single-center experience assessing risk factors and outcomes. Pediatr Transplant. 22(7): e13267.

Volpi S, Doenz F, Qanadli SD. 2018. Superior Vena Cava (SVC) Endovascular Reconstruction with Implanted Central Venous Catheter 
Repositioning for Treatment of Malignant SVC Obstruction. Front Surg. 5: 4 .

Wang ZS, Li CW, Li JX, Wu WJ, Li Y, Shi JG. 2020. Covered versus uncovered stent insertion for malignant superior vena cava obstruction. Minim Invasive Ther Allied Technol. 29(6): 353-8.
Xu YS, Fu YF, Du HT, Li DC. 2015. Palliative Stent Insertion for Acute Malignant Colorectal Obstruction: Long-term Patency and Survival. Surg Laparosc Endosc Percutan Tech. 25(6): 500-4.

Yuuki T, Kanda T, Kimura Y, et al. 2001. Inflammatory cytokines in vitreous fluid and serum of patients with diabetic vitreoretinopathy. J Diabetes Complications. 15(5): 257-9. 